\title{
A NOVEL INCREMENTAL CLUSTERING FOR INFORMATION EXTRACTION FROM SOCIAL NETWORKS
}

\author{
Bhavani Pappula ${ }^{1}$, Seetha Maddala ${ }^{2}$ \\ ${ }^{1} P G$ Scholar, Department of Computer Science and Engineering, G.Narayanamma Institute of Technology and \\ Science, Telangana, India \\ ${ }^{2}$ Professor \& HOD, Department of Computer Science and Engineering, G.Narayanamma Institute of Technology and \\ Science, Telangana, India
}

\begin{abstract}
The challenge of this project concentrates upon the issue of synopsis on the remark string regarding the particular message from social media. Because of the more fame of social media, amount of remarks may increment by the side of more ratio directly later the societal message is printed. Clients can want to achieve the detailed comprehension about remark string without study entire remark set, an attempt is made in order to bunch remarks by comparative substance all at once also produce the succinct judgment outline only for this message. Seeing that any time various clients can ask for a synopsis outcome, but the existing clustering strategies cannot fulfill the current requirement of this program. We design an incremental bunching issue for remark string synopsis upon social media also propose Incremental Clustering method it can incrementally bring up to date bunching outcomes including recent arriving remarks. And also, we design a presentation interface comprising of fundamental data, keyterms, and delegate remarks. This brief look presentation interface assists clients to rapidly achieve the outline comprehension about remark string. From the experimental results it is observed that Incremental Clustering method is more efficient than $K$ Means and Batch clustering methods.
\end{abstract}

Keywords: Clustering, Summarization, Remark Strings, SNS (Social Network Services).

\section{INTRODUCTION}

Of late, (SNS) are predominant and have ended up imperative correspondence frameworks inside our day by day life. Identifying with the 2012 figures by the most noteworthy agreeable systems administration site Facebook, there are more than 500 mil day by day dynamic users and a normal of 3: 2 billion communications are made every day. Furthermore, Micro-blogging goliath Twitter has more than 400 million client essential and there are close to 200 million messages distributed in one day. Because of the fame and simplicity of these stages, superstars, enterprises, and associations additionally fabricate interpersonal sheets to communicate along with their aficionados and the general population. Keep in mind it for every message, the people can tell their perspectives by sending, providing a like, and putting remarks for that.

In any case, we may even now try to truly recognize what are they discussing and what are the perspectives of those conversation members. Furthermore, famous people and partnerships will have high enthusiasm to see how their supporters and users responding to specific issues and substance. With these inspirations, we are inspirited to build up an expert outline method focusing on at remark strings in social media. Various reviews and programming has intended systems as well as procedures for making different sorts of outlines on remark strings. One particular major category plans to excerpt ideal and important remarks from messy discussion. Like Facebook [1] and YouTube [2], these famous administrations permit clients to figure out if a remark pays to or else advisable, also remarks which have recommendations with highest rank were shown in an upper part of the set. About the other hand, a few analysts design this issue as suggestion [3], [4], [5]or distribution errands in addition to resolve this issue they utilize natural language processing methods. Besides, emotion inquiry [6] has been used to find secret considerations in messages. Besides, giving a useful presentation interface [7] is another effective exploration field on the summary of societal messages.

\section{PROPOSED WORK}

In this paper, do not give attention to conventional remark strings that typically express more finish data, including the conversation on items or films. We objective at remark strings in social media with regular dialect utilization in a brief content manner. On behalf of every societal message, bunch remarks by comparative substance all at once also produce the succinct judgment outline only for this message is the foremost goal. We need to find how various bunch suppositions exist and offer a review of every bunch to make users effortlessly and quickly get it. To get case, when Lady Decrepito transfers a picture to social media, there are a lot of remarks given by her fans amid a brief time. Some of them may say that the young lady with exceptionally wonderful, and another bunch of aficionados may feel that the ensemble is excessively odd. Potentially all the more, some may particularly examine the haircut of this picture. Therefore, our objective is quick building up a productive and effectual strategy to recognize the groups of these remarks. 
Be aware that this issue is apparently entirely distinct from existing exploration and has various exceptional qualities and difficulties. Starting, the amount of remarks may increment by the side of more ratio directly later the societal message is printed. Besides, unmistakable any time clients can ask for a synopsis outcome. To satisfy the convenient requirement of this program, consequently, so as to quickly make an outline taking into account the present remark string, an incremental methodology is more appropriate. Besides, remarks within social media are typically concise, and clients generally utilize casual and unstructured content informing that contain acronyms, diminishing words, and so on. This occurrence builds the trouble of choosing the likeness between remarks.

Then again, this truly valuation saying that rather than focusing upon standard of bunching, ultimate important statement for such errand is towards deliver a regular synopsis rapidly therefore clients can effectively achieve an outline regarding the remark string. This is typically observed that this issue is equipped for being designed as a bunching procedure. Notwithstanding, conventional clustering strategies have a few intrinsic imperatives that cannot be straight connected here. In the first place, existing strategies intricacy is more, in addition existing strategies cannot directly adjust to gratify an incremental necessity. Additionally, in this issue, characterizing the amount of wanted groupings ahead of time is not logical, which is required in numerous bunching methods. Additionally, there will be a bulk of exceptions in a remark string, which implies that without making utilization of a decent technique for selecting first group focuses, existing strategy might be inclined to poor results.

\subsection{Batch Clustering}

Accordance with the issue definition, we propose the method batch clustering that is the batch version for resolving this issue. Batch Clustering takes the entire brief remark list L is as an input. Next sort is threshold utilized in consideration of deciding in what way remarks within a group are comparable. By and large there are two fundamental strides in Batch Clustering. The goal of the initial step is to locate every single related segment of the remark list L. However the details related to the similar linked part will be converged as a bunch. As it may be envisioned that there will be a connection among binary remarks while their separation cannot be unbounded.

To get every remark of L, we inspect whether there is positively any actual bunch where the distance between remark and bunch is not infinite. In the event that there is, this remark will be included into anybody of these bunches. Something else, another single-point group is made with the remark. We estimate the separations among binary focuses of some couple of bunches which has more than one statement, obviously, in case that separation among two bunches cannot be boundless, and then they might be blended as a group. Afterwards, the second principal stage goal is that ensure the span related to every bunch is littler than the threshold. In order to address such need, in consideration of every more than one statement bunch, we remove the remark where the distance is bigger than or equivalent to threshold. In the interim, it can be checked whether remark can be mixed with other prohibited remarks. After this progression, all bunches will meet the sweep confinement, lastly, Batch Clustering yields the top-k groups with top-k best remarks.

\subsection{Incremental Clustering}

Because of the more fame of social media, amount of remarks related to the particular message can increment rapidly, as well as clients can demand for the purpose of seeing a synopsis about remarks whenever. Also, since new messages show up reliably, clients for the most part just view the synopsis of a particular message once and will not retreat again to take a gander at redesigned brief synopsis in the future. In such framework, to promptly create the most recent top-k groups, an Incremental Clustering method is proposed which gets a capacity regarding incremental reexamine. An incremental Clustering method basic idea is that keep up bunching response to the past stage, additionally to incrementally bring up to date grouping effect with the recently arriving remark.

\subsection{Presentation Interface}

Taking after top-k bunches are made, following point that the manner by which to show the synopsis outcomes. Upon social media by using blasting quantity of data, our goal is that give a compact also a brief look presentation interface which grants clients to rapidly achieve the outline comprehension about remark string. In this manner, two sorts of synopsis are considered. For every bunch, an arrangement of delegate key-terms that are much of the time said will be excerpted for developing a key-term cloud. Besides, a few delegate remarks will also be recognized.

\section{RESULTS AND DISCUSSIONS}

Different results are shown below. We collected remark strings from the facebook graph $\mathrm{API}^{3}$. Through the highest facebook sheets in January 2016, we chosen 5 of them. The performance of the clustering methods is evaluated based on their efficiency in terms of computing time taken by each clustering method. The performance of the clustering methods is also evaluated by using precision, recall, f-score parameters. The below Table-1 summarizes the information about the total comments in each category of the datasets.

Table -1: Number of Comments in each Category

\begin{tabular}{|l|l|l|}
\hline Category & Comments & Total \\
\hline Sports & ViratKohli & 185 \\
\hline \multirow{2}{*}{ Politics } & Kejrival & 735 \\
\cline { 2 - 3 } & Rahul Gandhi & 2151 \\
\hline Health & Ramdev & 402 \\
\hline Business & Finance Minister & 600 \\
\hline
\end{tabular}

The below Table- 2 presents the computing time taken by each clustering method i.e. K-Means, Batch Clustering and Incremental Clustering for various comments datasets. 
Table -2: Execution Time taken by K-Means, Batch and Incremental Clustering methods for various datasets

\begin{tabular}{|l|l|l|l|l|l|}
\hline Clustering & \multicolumn{6}{|l|}{ Execution Time (milli seconds) } \\
\hline & $\begin{array}{l}\text { Virat Kohli } \\
\text { (Dataset 1) }\end{array}$ & $\begin{array}{l}\text { Kejrival } \\
\text { (Dataset2) }\end{array}$ & $\begin{array}{l}\text { Ramdev } \\
\text { (Dataset3) }\end{array}$ & $\begin{array}{l}\text { Finance Minister } \\
\text { (Dataset 4) }\end{array}$ & $\begin{array}{l}\text { Rahul Gandhi } \\
\text { (Dataset5) }\end{array}$ \\
\hline K-Means & 7500 & 145023 & 44346 & 88567 & $8,50,000$ \\
\hline Batch & 4328 & 84974 & 31580 & 60425 & $6,90,000$ \\
\hline Incremental & 3922 & 72176 & 29158 & 57737 & $5,50,000$ \\
\hline
\end{tabular}

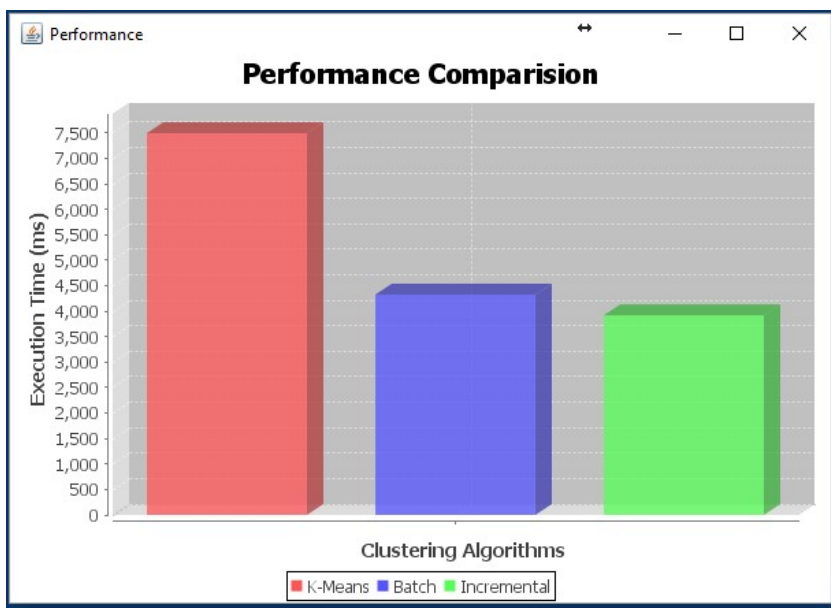

Fig-1: Execution Time Graph for ViratKohli Comments (Dataset-1)

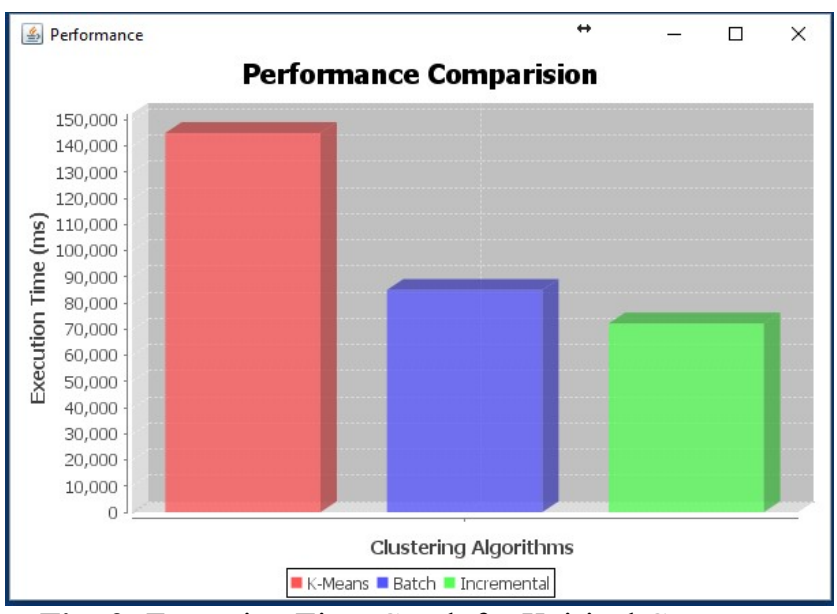

Fig -2: Execution Time Graph for Kejrival Comments (Dataset-2)

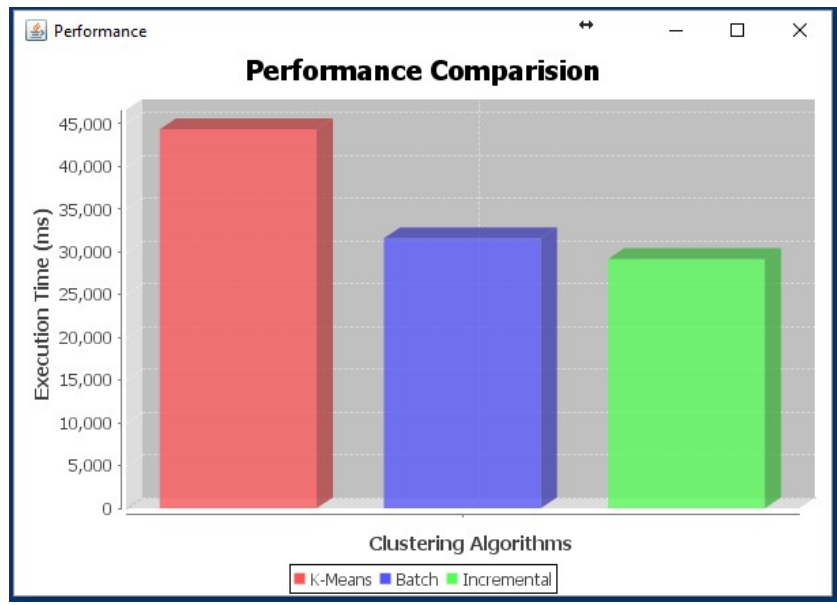

Fig -3: Execution Time Graph for Ramdev Comments (Dataset-3)

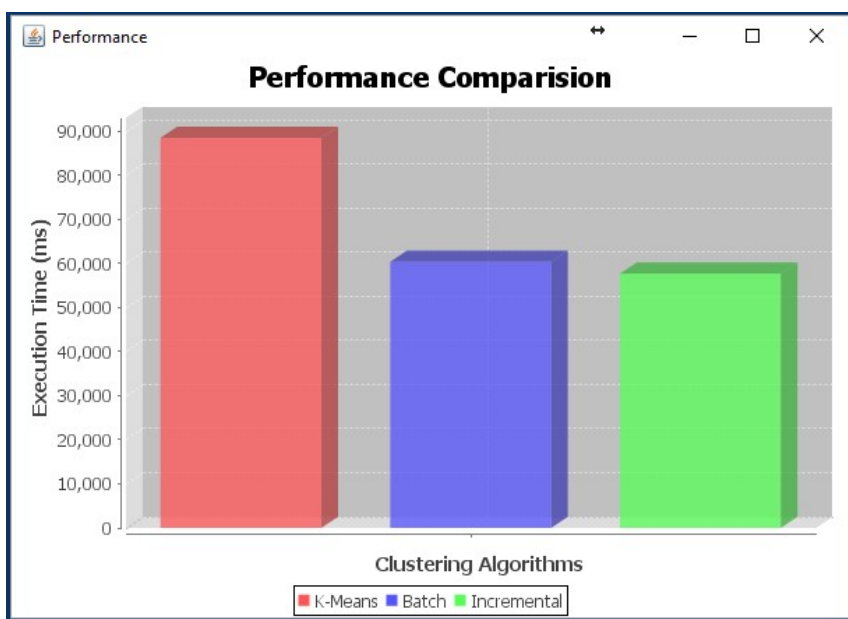

Fig -4: Execution Time Graph for Finance Minister Comments (Dataset-4)

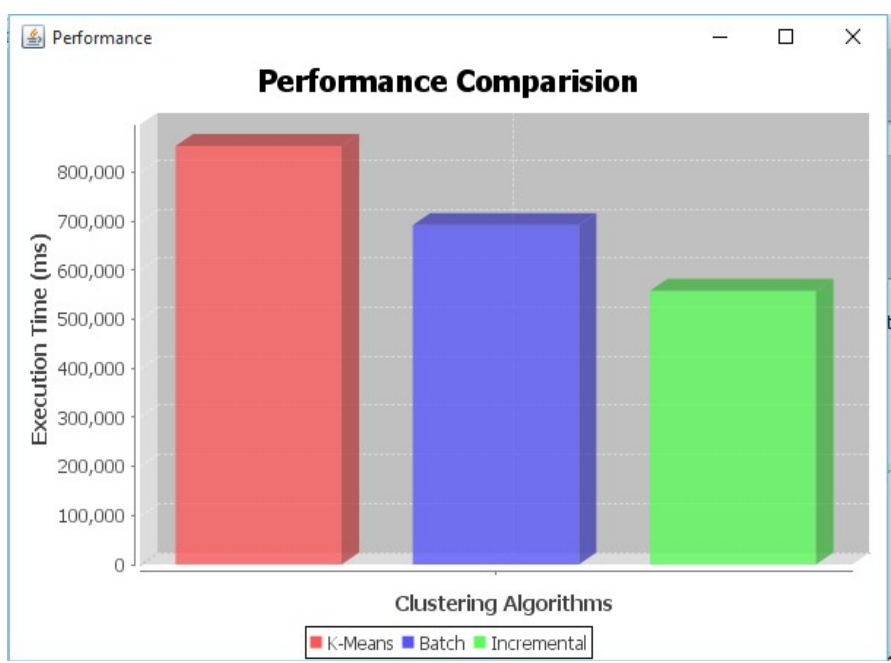

Fig -5: Execution Time Graph for Rahul Gandhi Comments (Dataset-5)

From the above graphs (Fig-1 to Fig-5) it is observed that Incremental Clustering takes less time compared to Batch and K-Means clustering methods. Therefore an Incremental Clustering method is more efficient than other two clustering methods. The performance of Incremental Clustering method is better than other clustering methods. The performance of the clustering methods is also evaluated based on precision, recall and f-score on the K-Means, Batch Clustering and Incremental Clustering methods. The below Tables (Table-3 to Table-5) and Charts (Chart-1 to Chart-3)presents the precision, recall, and f-score computed for each clustering method i.e. K-Means, Batch Clustering and Incremental Clustering methods for various comments datasets. 
Table -3: Precision for various datasets

\begin{tabular}{|l|l|l|l|}
\hline \multirow{2}{*}{ Datasets } & K-Means & Batch & Incremental \\
\cline { 2 - 4 } & Precision & Precision & Precision \\
\hline Dataset1 & 64.45 & 78.27 & 90.09 \\
\hline Dataset2 & 60.52 & 80.52 & 84.52 \\
\hline Dataset3 & 61.34 & 74.83 & 78.85 \\
\hline Dataset4 & 70.58 & 79.35 & 82.76 \\
\hline Dataset5 & 60.68 & 62.48 & 70.56 \\
\hline
\end{tabular}

The above Table-3 presents the Precision computed for each clustering method i.e.K-Means, Batch Clustering and Incremental Clustering methods.

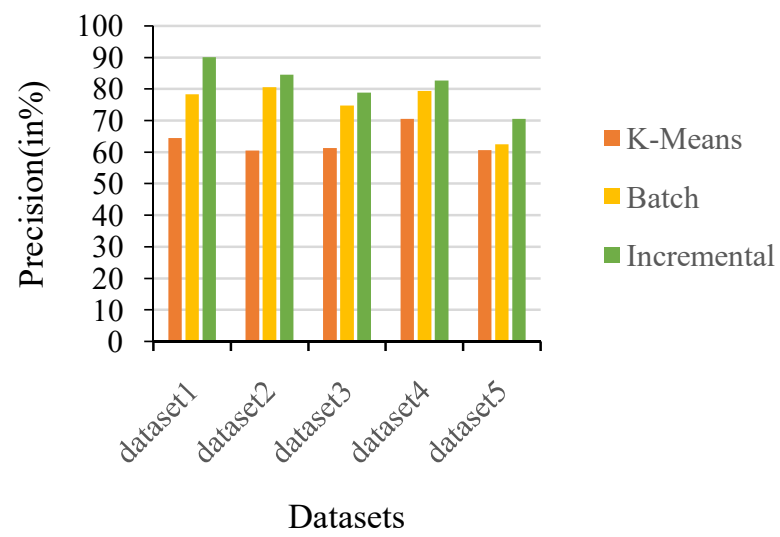

Chart -1: Precision Graph for various datasets

The below Table-4 presents the Recall computed for each clustering method i.e.K-Means, Batch Clustering and Incremental Clustering methods.

Table -4: Recall for various datasets

\begin{tabular}{|l|l|l|l|}
\hline \multirow{2}{*}{ Datasets } & K-Means & Batch & Incremental \\
\cline { 2 - 4 } & Recall & Recall & Recall \\
\hline Dataset1 & 58.85 & 63.27 & 68.29 \\
\hline Dataset2 & 56.23 & 65.36 & 69.86 \\
\hline Dataset3 & 59.37 & 60.45 & 70.23 \\
\hline Dataset4 & 60.86 & 68.29 & 73.65 \\
\hline Dataset5 & 55.28 & 58.49 & 64.46 \\
\hline
\end{tabular}

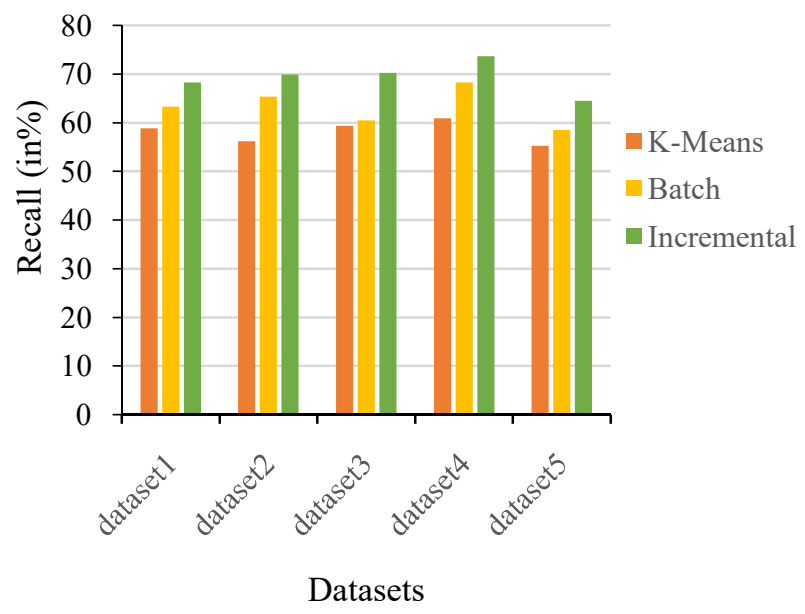

Chart -2: Recall Graph for various datasets
The below Table-5 presents the F-Score computed for each clustering method i.e.K-Means, Batch Clustering and Incremental Clustering methods.

Table -5: F-Scorefor various datasets

\begin{tabular}{|l|l|l|l|}
\hline \multirow{2}{*}{ Datasets } & K-Means & Batch & Incremental \\
\cline { 2 - 4 } & F-Score & F-Score & F-Score \\
\hline Dataset1 & 61.52 & 69.98 & 77.69 \\
\hline Dataset2 & 58.29 & 72.15 & 76.50 \\
\hline Dataset3 & 60.34 & 66.87 & 74.30 \\
\hline Dataset4 & 65.36 & 73.40 & 77.94 \\
\hline Dataset5 & 57.85 & 60.42 & 67.37 \\
\hline
\end{tabular}

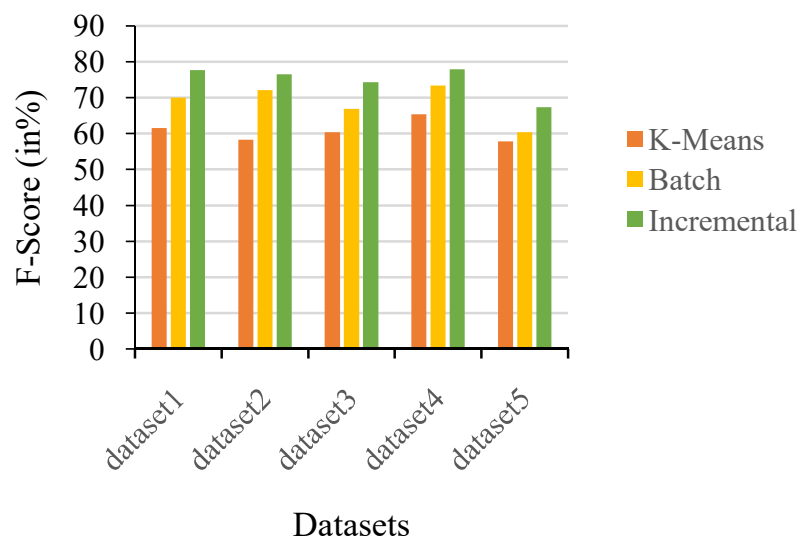

Chart -3: F-Score Graph for various Datasets

The analysis is performed using the above parameters i.e. precision, recall and f-score. The comparison between $\mathrm{K}$ Means, Batch and Incremental clustering methods is performed for precision, recall and f-score. From the chart-1 to chart-3 it is observed that in chart-1 Precision is more for Incremental Clustering in every dataset compared to $\mathrm{K}$ Means and Batch clustering methods and in chart-2 Recall is more for Incremental Clustering in every dataset compared to K-Means and Batch clustering methods and in chart-3 FScore is more for Incremental Clustering in every dataset compared to K-Means and Batch clustering methods.

\section{CONCLUSION}

To allow the capacity of remark string synopsis upon social media, a new incremental bunching issue is designed also an Incremental Clustering method is proposed, it will incrementally bring up to date bunching results with the most recent arriving remarks progressively. With the result of Incremental Clustering, we model a presentation interface comprising of fundamental data, key-terms, and delegate remarks. This brief look presentation interface grants users to rapidly achieve the outline comprehension of a remark string. The performance of the clustering methods is evaluated based on their efficiency in terms of computing time taken by each clustering method. The performance of the clustering methods is also evaluated based on precision, recall and f-score parameters. From the experimental results it is observed that Incremental Clustering method is more efficient than K-Means and Batch clustering methods. 


\section{REFERENCES}

[1]. Facebook [Online]. Available: http://www.facebook.com/, 2014.

[2].YouTube [Online]. Available: http://www.youtube.com/, 2014.

[3]. E. Khabiri, J. Caverlee, and C.-F. Hsu, "Summarizing UserContributed Comments," in Proc. 5th Int. AAAI Conf. Weblogs Soc. Media, 2011, pp. 534-537.

[4]. H. Becker, M. Naaman, and L. Gravano, "Selecting quality twitter content for events," in Proc. 5th Int. AAAI Conf. Weblogs Social Media, 2011, pp. 442-445.

[5]. D. Chakrabarti and K. Punera, "Event summarization using tweets," in Proc. 5th Int. AAAI Conf. Weblogs Social Media, 2011, pp. 66-73.

[6]. J. Bollen, H. Mao, and A. Pepe, "Modeling public mood and emotion: Twitter sentiment and socio-economic phenomena," in Proc. 5th Int. AAAI Conf. Weblogs Social Media, 2011, pp. 450-453.

[7]. A. Marcus, M. S. Bernstein, O. Badar, D. R. Karger, S. Madden, and R. C. Miller, "TwitInfo: Aggregating and visualizing microblogs for event exploration," in Proc. ACM SIGCHI Conf. Human Factors Comput. Syst., 2011, pp. 227-236.

[8]. Cheng-Ying Liu, Ming-Syan Chen, Fellow, IEEE, and Chi-Yao Tseng, "IncreSTS: Towards Real-Time Incremental Short Text Summarization on Comment Streams from Social Network services", in IEEE Trans. Knowl. Data Eng., Vol. 27, No. 11, pp. 2986-3000, November 2015.

\section{BIOGRAPHIES}

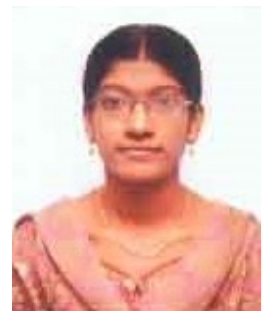

P.Bhavani is pursuing her M.Tech in Computer Science and Engineering (CSE) from G.Narayanamma Institute of Technology and Science, Hyderabad, and completed her B.Tech in Computer Science and Engineering from Jyothishmathi Institute of Technology and Science, Karimnagar in the year 2013. Her research areas of interest includes data and text mining.

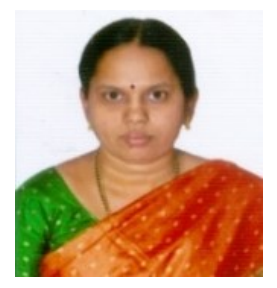

Dr. M.Seetha had completed Ph.D in Computer Science and Engineering in the area of image processing in December 2007 from Jawaharlal Nehru Technological University, Hyderabad, India. She is presently working as Professor\& HOD in Department of CSE in GNITS, Hyderabad and has the teaching experience of 22 years. She is guiding $8 \mathrm{Ph} . \mathrm{D}$ scholars and 2 research scholars completed Ph.D under her guidance. Her research interest includes image processing, neural networks, computer networks and data mining. She had published more than 70 papers in refereed journals and 90 in the proceedings of National/International Conferences and Symposiums. 